\title{
Collective dynamics of simple liquids: A mode-coupling description
}

\author{
W.Schirmacher ${ }^{1,2}$, H.Sinn ${ }^{1,3}$ \\ ${ }^{1}$ Argonne National Laboratory, 9700 S. Cass Avenue, Argonne, IL 60439, USA \\ 2 Physik-Department E13, Technische Universität München, James-Franck-Strasse 1, D-85747 Garching, \\ Germany \\ 3 Hasylab/DESY, Notkestrasse 85, D-22607 Hamburg, Germany
}

Received November 27, 2007, in final form - February 12, 2008

\begin{abstract}
We use the mode-coupling theory (MCT), which has been highly successful in accounting for the anomalous relaxation behaviour near the liquid-to-glass transition, for describing the dynamics of monatomic (i.e. simple) liquids away from the glass formation regime. We find that the dynamical structure factor predicted by MCT compares well to experimental findings and results of computer simulations. The memory function exhibits a two-step decay as found frequently in experimental and simulation data. The long-time relaxation regime, in which the relaxation rate strongly depends on the density and is identified as the $\alpha$ relaxation. At high density this process leads the glass instability. The short-time relaxation rate is fairly independent of density.
\end{abstract}

Key words: liquid dynamics, collective excitations in liquids, simple liquids, mode-coupling theory

PACS: $62.60 .+V, 66.20 .-d$

\section{Introduction}

To find a theoretical description of the dynamics of simple monatomic liquids in the microscopic frequency and wavenumber regime (involving inverse Angstroms and inverse picoseconds), which is accessible to inelastic neutron and X-ray scattering, is already a long-standing issue [1-5]. One of the important quantities of interest is the dynamical structure factor $S(q, \omega)$, which is the spaceand-time Fourier transform of the density-density correlation function. $S(q, \omega)$ is proportional to the coherent inelastic scattering cross-section of neutrons and X-rays. It contains information on sound-like collective excitations in the liquid. As the static physical quantities and correlation functions can be calculated without knowledge of the dynamics in classical liquids, the static and dynamical properties are a separate issue. The former serve as input quantities if one is interested in the latter. A simple liquid is usually defined to be a monatomic one if it can be described by a pairwise potential. Given such a potential the static structure factor $S(q)=\int_{-\infty}^{\infty} \mathrm{d} \omega S(q, \omega)$ ("zeroth moment") can be calculated by the Monte-Carlo (MC) method, or, approximately, by integral equation theories [2-4].

The static structure factor of simple liquids like liquid rare gases or liquid metals has turned out to be dominated by the short-range repulsive part of the potential [3,4]. Therefore in most cases $S(q)$ can essentially be described in terms of the structure factor of a hard-sphere fluid as calculated e.g. in Percus-Yevick (PY) approximation [6]. Efforts were made to describe the liquid dynamics as well in terms of that of the hard-sphere liquid, using generalizations of Enskog's kinetic theory $[3,4,7]$. The single-particle (incoherent) dynamics of liquid metals could have accounted for a successful combination of Enskog theory and molecular-dynamics results [8]. There are also attempts to describe the collective dynamics in terms of the hard-sphere dynamics [9]. However, the clearest and most successful description of the collective dynamics of simple liquids is the generalized hydrodynamics of Mori and Zwanzig [3,4,10]. This is so, because within such a description the moment sum rules, which follow from the conservation laws, are obeyed automatically. It is widely 
believed [1-4,11] that the frequency moments $\left.c_{q}^{(n)}=\int_{-\infty}^{\infty} \mathrm{d} \omega \omega^{n} S(q, \omega)\right)$ of $S(q, \omega)$, essentially determine the dispersion of the collective vibrational excitations of the liquid. The formalism is called generalized hydrodynamics because for small energies and momenta it goes over into linearized hydrodynamics [2-4]. In the general theory retardation effects are taken into account by memory functions $M_{q}(t)$, which replace the hydrodynamic friction coefficients. The task of a microscopic theory is to construct such functions.

Within the memory function formalism, the correlation functions of the collective modes obey generalized damped-oscillator equations (generalized Langevin equations) with the damping term replaced by a convolution of $M_{q}(t)$ with the time derivative of a correlation function. This corresponds to a frequency dependent friction term in the frequency domain.

The theoretical approaches used so far for the description of the dynamics of simple liquids in terms of such memory functions differ in the kind of collective modes that were treated. The simplest way is to consider density fluctuations only. In fact $S(q, \omega)$ is just the Fourier transform of the density-density correlation function $F_{q}(t)$. A simple approach was to use a phenomenological ansatz $M_{q}(t) \propto \mathrm{e}^{-t / \tau_{q}}$ [1] and to fit the function $\tau_{q}$ to the experimental or simulational data. However, such a single-exponential ansatz was already shown to be insufficient to fit early simulation data of Lennard-Jones Argon [12] in the early days of this subject. Levesque et al. [12] obtained evidence for a second, rather slow decay of the memory function. Such a behaviour of $M_{q}(t)$ has been confirmed more recently in the analysis of inelastic X-Ray scattering data of liquid metals $[5,13-15]$ as well as pertinent simulations [5,16]. Although finding an appropriate fit function for the memory function is an efficient way to represent data on $S(q, \omega)$ (see, however, [17] for another efficient procedure) it does not comprise a theory, except for the evaluation of the moments. A complete theory is obtained if the memory function is predicted by the theory as well. This is the case for the different versions of mode-coupling theory. In the density fluctuation mode-coupling theory $(\mathrm{MCT}) M_{q}(t)$ is approximated as a linear combination of products of density correlation functions. This theory was first used for the excitation spectrum of liquid Helium[18]. Its classical version [19-22] is the basis of the very successful MCT description of the liquid-to-glass transition and the non-trivial scaling relations near the transition. MCT has been also shown to describe the salient features of the vibrational dynamics in the glassy state $[23,24]$.

In order to study the dynamics of simple classical liquids, the current fluctuation version of mode-coupling theory [25] has been utilized. In this version, the memory functions, which appear in the generalized Langevin equations of the longitudinal and transverse current fluctuations, are approximated as a linear combination of products of current and density correlation functions. Although this theory has been successfully used to account for the salient features of some simple liquids, a drawback is that it involves a rather complicated set of equations with vertex functions which can only be calculated if the underlying potential is known. This is not the case for the density version of MCT, where only $S(q)$ and some properties like density and temperature need to be known as input of the theory. Quite recently it has been shown that this version of MCT is capable of successfully describing the collective dynamics of liquid titanium above the melting point [26]. The only input was the experimentally measured structure factor. A schematic version of MCT has been shown to accurately describe light scattering spectra on liquid benzene away from the glass transition [27].

In what follows we shall explore the predictions of MCT using a hard-sphere like model structure factor in which we vary the packing fraction $\eta$ ("density") as the only material parameter. We find that the essential features of the collective excitations of simple liquids in the density range below the glass transition can be described in terms of MCT. In particular, we find for the memory function $M_{q}(t)$ that it exhibits a two-step decay. The first takes place on a microscopic time scale and only weakly depends on the density. The second is an exponential decrease with a relaxation time which is much larger than the first one and strongly increases with density. We identify this relaxation process as the structural ("alpha") relaxation, which is governed by the so-called cage effect. The latter term denotes the fact that in a dense liquid, a particle cannot move without the adjacent ones, which form a cage around a given particle. 


\section{Formalism}

Within the MCT the dynamical structure factor $S(q, \omega)$, which is proportional to the inelastic coherent neutron or inelastic ionic X-ray scattering cross-section is expressed in terms of the density memory function $M_{q}(t)$, or its Fourier-Laplace transform [28] $M_{q}(\omega)=\int_{0}^{\infty} \mathrm{d} t \mathrm{e}^{-\mathrm{i} \omega t} M_{q}(t)$ as

$$
S(q, \omega)=\frac{1}{2 \pi} \int_{-\infty}^{\infty} \mathrm{d} t F_{q}(t) \mathrm{e}^{-\mathrm{i} \omega t}=\frac{1}{\pi} \operatorname{Re}\left\{\frac{S(q)}{\mathrm{i} \omega+\frac{\Omega_{0}^{2}(q)}{\mathrm{i} \omega+M_{q}(\omega)}}\right\} .
$$

In the time representation this expression can be cast into the following generalized harmonic oscillator equation ("generalized Langevin equation" $[3,4,10]$ )

$$
\ddot{F}_{q}(t)+\int_{0}^{t} \mathrm{~d} \tau M_{q}(t-\tau) \dot{F}_{q}(\tau)+\Omega_{0}^{2}(q) F_{q}(t)=0 .
$$

with the isothermal sound dispersion

$$
\Omega_{0}^{2}(q)=v_{\mathrm{th}}^{2} q^{2} / S(q)=c_{2} / c_{0},
$$

where $v_{\text {th }}$ is the thermal velocity defined by $v_{\text {th }}^{2}=k_{\mathrm{B}} T / m$. As stated above, the formulation in terms of a memory function guarantees that the first two sum-rule requirements, namely those of the 0 -th and the second moment $c_{0}$ and $c_{2}$ are fulfilled. In the density fluctuation version of the mode-coupling theory $(\mathrm{MCT})[19,21,23] M_{q}(t)$ is self-consistently related to $F_{q}(t)$ in the following way:

$$
M_{q}(t)=\Omega_{0}^{2}(q) \frac{1}{2 V} \sum_{\mathbf{q}_{1}, \mathbf{q}_{2}}^{\mathbf{q}_{1}+\mathbf{q}_{2}=\mathbf{q}} V_{\mathbf{q q}_{1} \mathbf{q}_{2}} F_{q_{1}}(t) F_{q_{2}}(t)
$$

Here $V$ is the total volume. For the evaluation of the sum over $\mathbf{q}_{1}$ and $\mathbf{q}_{2}$ see the appendix. The vertex (in a version where the three-body static correlation functions are decoupled by the superposition approximation, see $[19,21])$ is given by

$$
V_{\mathbf{q q}_{1} \mathbf{q}_{2}}=\frac{1}{n} S(q) W_{\mathbf{q q}_{1} \mathbf{q}_{2}}^{2},
$$

where $n=N / V$ is the particle number per volume,

$$
W_{\mathbf{q q}_{1} \mathbf{q}_{2}}=\frac{1}{q^{2}} \mathbf{q} \cdot\left[\mathbf{q}_{1} n c\left(q_{1}\right)+\mathbf{q}_{2} n c\left(q_{2}\right)\right],
$$

where

$$
c(q)=\frac{1}{n}\left(1-\frac{1}{S(q)}\right)
$$

is the direct correlation function.

Equations (2) and (4) are a closed set of equations for $F_{q}(t)$ which can be solved on a computer with the initial conditions $F_{q}(0)=S(q)$ and $\dot{F}_{q}(0)=0$.

\section{Structure factor}

As mentioned in the introduction, the static structure factors of most simple liquids, especially those of liquid metals, can be well described in terms of the hard-sphere static structure factor in Percus-Yevick (HS-PY) approximation. However, at wavenumbers larger than the principal peak of $S(q)$ the HS-PY structure factor does not decay as rapidly towards the uncorrelated value $S(q)=1$ as the experimental data. This corresponds to the fact that the radial pair distribution function 
$g(r)=1+n \int \frac{\mathrm{d}_{\mathbf{q}}}{2 \pi} \mathrm{e}^{\mathrm{i} \mathbf{q r}}[S(q)-1]$ in PY approximation abruptly jumps from 0 to its maximum value at $r=\sigma$, where $\sigma$ is the hard-sphere diameter. This is, of course, not the case for simple liquids, in which $g(r)$ rises continuously in a smooth way towards its maximum at a value of $r$, which is slightly larger than $\sigma$. We found that the experimentally measured $S(q)$ data of several liquid metals can be quite satisfactorily described in the total $q$ range by the formula

$$
S(q)=1+\left[S_{\mathrm{HS}}(q)-1\right] \mathrm{e}^{-\frac{1}{2}(\lambda q \sigma)^{2}}
$$

with $\lambda \approx 0.05$, where $S_{\mathrm{HS}}(q)$ is the HS-PY structure factor (figure 1a). This corresponds to a convolution of $g_{\mathrm{HS}}(r)-1$ with a Gaussian of width $\sigma \lambda$.

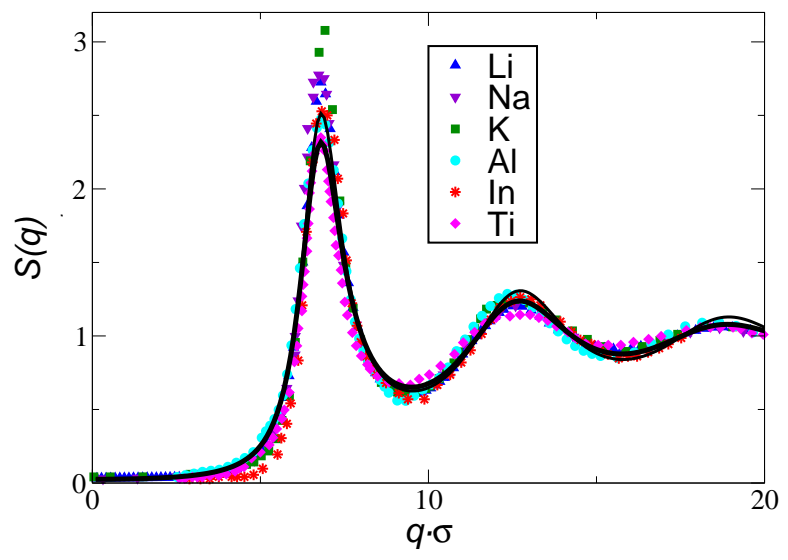

Figure 1. Static structure factors of several liquid metals. Thin black line: Hard-Sphere PY structure factor, thick black line: equation (8), both calculated with packing fraction $\eta=0.45$. References for the experimental data can be found in [5].

There is another, in fact, more important reason for using (8) instead of the HS-PY structure factor. Using the hard-sphere structure factor for calculating the memory function in (4) (or (A4), resp.) leads to a vertex function $V_{\mathbf{q q}_{1} \mathbf{q}_{2}}$ which decays very slowly for large wavenumbers $Q=$ $\frac{1}{2}\left|\mathbf{q}_{1}-\mathbf{q}_{2}\right|$, so that there are convergence problems in the numerics.

\section{Results and discussion}

We have solved equations (2) and (4) numerically, using structure factors given by (8) as input. These depend on two parameters, namely the decay constant $\lambda$, which we set equal to 0.05 throughout, and the packing fraction

$$
\eta=\frac{\pi}{6} n \sigma^{3}
$$

which controls the density dependence. We used $\sigma$ as length scale and the isothermal velocity $v_{\mathrm{T}}$ as frequency scale, divided by $\sigma$ :

$$
\omega_{0}=\frac{v_{\mathrm{T}}}{\sigma}=\frac{v_{\mathrm{th}}}{\sigma \sqrt{S(q=0)}}=\frac{1}{\sigma} \sqrt{\frac{k_{\mathrm{B}} T}{m S(q=0)}}
$$

$v_{\mathrm{T}}$ can be obtained either from the measured ultrasonic sound velocity $v_{\mathrm{S}}=v_{\mathrm{T}} / \kappa$ (where $\kappa$ is the ratio of the specific heats at constant pressure and volume) or from $S(q=0)$. $\kappa$ is approximately unity in the $\mathrm{ps}^{-1}$ frequency regime (see, however, the table given in [5]). In these units $\lambda$ and $\eta$ are the only parameters of the theory. The temperature enters solely through $\eta$. It should be noted that in these units the isothermal sound velocity is fixed to unity.

In figure $2 \mathrm{a}$ we have plotted the normalized dynamical structure factor $\tilde{S}(q, \omega)=S(q, \omega) / S(q)$ for 10 representative wavenumbers. This quantity is dominated by the central peak, which describes 

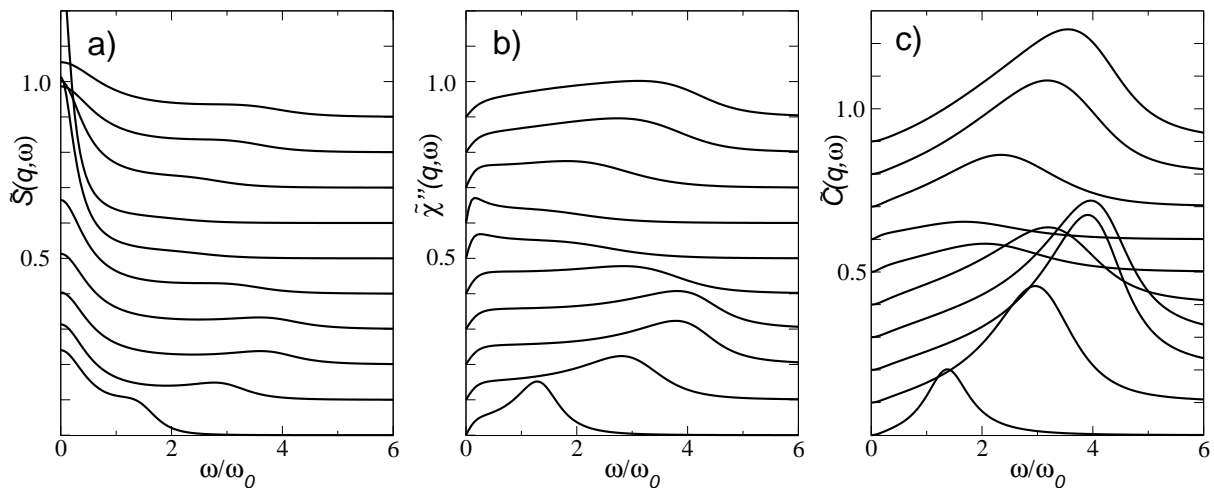

Figure 2. $\tilde{S}(q, \omega) \equiv S(q, \omega) / S(q), \tilde{\chi}^{\prime \prime}(q, \omega) \equiv \omega \tilde{S}(q, \omega)$ and $\tilde{C}(q, \omega) \equiv \omega^{2} \tilde{S}(q, \omega)$ for $\eta=0.45$ and for (from bottom to top) $q \cdot \sigma=1,2, \ldots 10$. The curves for $q \cdot \sigma>1$ have been moved up by integer amounts of 0.1 for better visibility.

the relaxation dynamics of the liquid. At small momentum transfer $\tilde{S}(q, \omega)$ has side maxima, which correspond to the collective excitations. These maxima become more distinct in the quantities $\tilde{\chi}^{\prime \prime}(q, \omega)=\omega \tilde{S}(q, \omega)$ (out-of-phase dynamic susceptibility) and $\tilde{C}(q, \omega)=\omega^{2} \tilde{S}(q, \omega)\left(q^{2}\right.$ times the longitudinal current correlation function [2-4]). In figure $3 \mathrm{a}$ we have plotted the maxima and line widths (HWHM) of $\tilde{C}(q, \omega)$ vs. $q \sigma$ for several values of $\eta$. It is remarkable that there is no change in the generalized sound dispersion $\Omega(q)=\max \{\tilde{C}(q, \omega)\}$ as $\eta$ is varied towards its critical value $\eta_{c} \approx 0.55$, where the glass transition occurs [19,21]. A similar behaviour is observed for the linewidth. This weak density dependence of the collective excitations, predicted by MCT remains to be verified by experiments and/or simulations. It means that the longitudinal collective excitations of the liquid are essentially the same as those of the frozen material. An interesting feature of the low- $q$ dispersion is the slight increase of the differential sound velocity $\partial \Omega / \partial q$ with $q$. Such an increase is observed not only in many liquid metals (see below) but also in many glasses [23,29]. This feature is produced by $M_{q}^{\prime \prime}(\omega)$, the reactive part of the memory kernel, which renormalizes the dispersion. The weak density dependence of the liquid dispersion $\Omega(q)$ is in strong contrast to
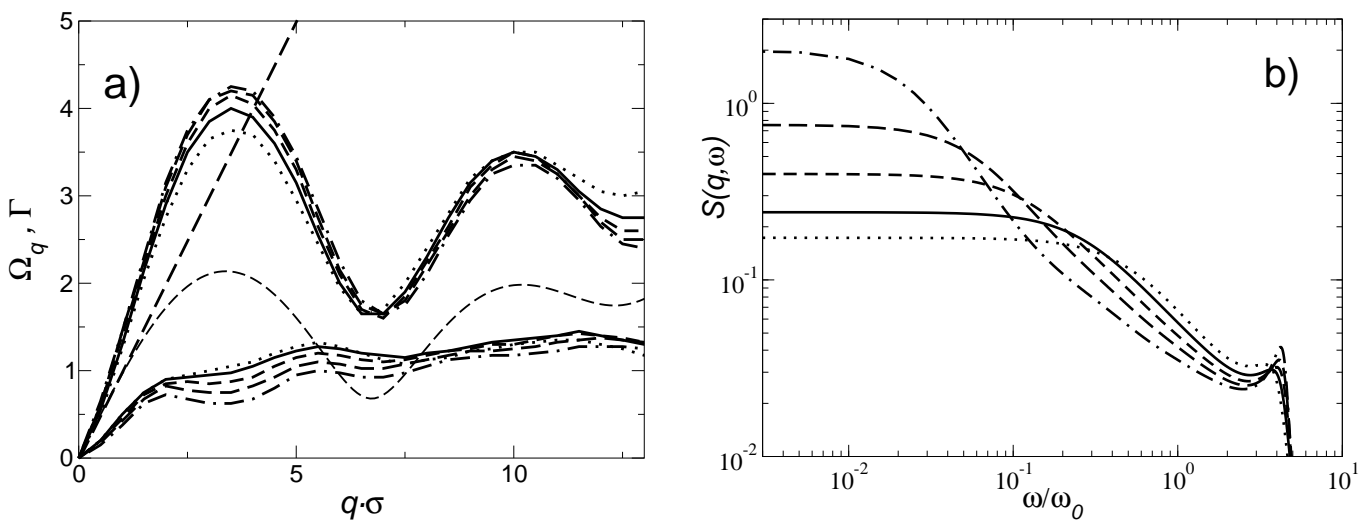

Figure 3. a: Dispersion and half widths at half maximum of the current correlation functions for $\eta=0.40$ (dots), 0.45 (straight line), 0.49 (short dashes), 0.52 (long dashes), 0.55 (dashdots). The lower thin dashed line is $\Omega_{q}$ as given in equation (3). The straight dashed line is the isothermal sound dispersion with $v_{i s o}=v_{\mathrm{th}} / \sqrt{S(q=0)}$. b: $\tilde{S}(q, \omega)$ for $q \sigma=0.35$ and the same densities.

the behaviour of the central line. The strong density change of the central maximum of $\tilde{S}(q, \omega)$ is most clearly seen in the double-logarithmic representation of figure 3b. The origin of this strong density variation can be understood if one realizes that the quantity $\tilde{S}(q, \omega=0)$ is proportional 
[30] to the so-called longitudinal $q$-dependent density

$$
\tilde{\eta}_{\ell}(q)=\frac{1}{\pi \rho k_{\mathrm{B}} T} \eta_{\ell}(q)=S(q, \omega=0) / S(q)^{2}=\tilde{S}(q, \omega=0) / S(q)
$$

For $q=0$ we have

$$
\eta_{\ell}(q=0)=\eta_{\mathrm{v}}+\frac{4}{3} \eta_{\mathrm{s}}
$$

where $\eta_{\mathrm{s}}$ is the usual shear density and $\eta_{\mathrm{v}}$ is the bulk or volume density.

As can be seen from figure 4 this quantity varies strongly with density, as expected, because it is this quantity which diverges on solidification. So we note that the central line - which is associated with the viscous flow - strongly depends on density, whereas the collective excitation frequencies and their line widths do not.

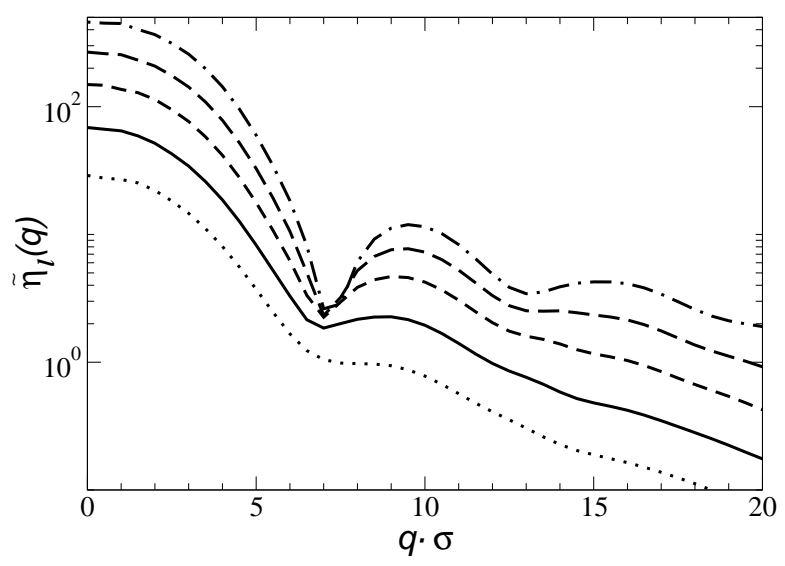

Figure 4. Viscosity $\tilde{\eta}_{\ell}(q) / n m$ as defined in the text for several densities. Line code as in figure 3.

Let us now have a look at the memory functions $M_{q}(t)$, which describe the relaxation processes in the liquid.

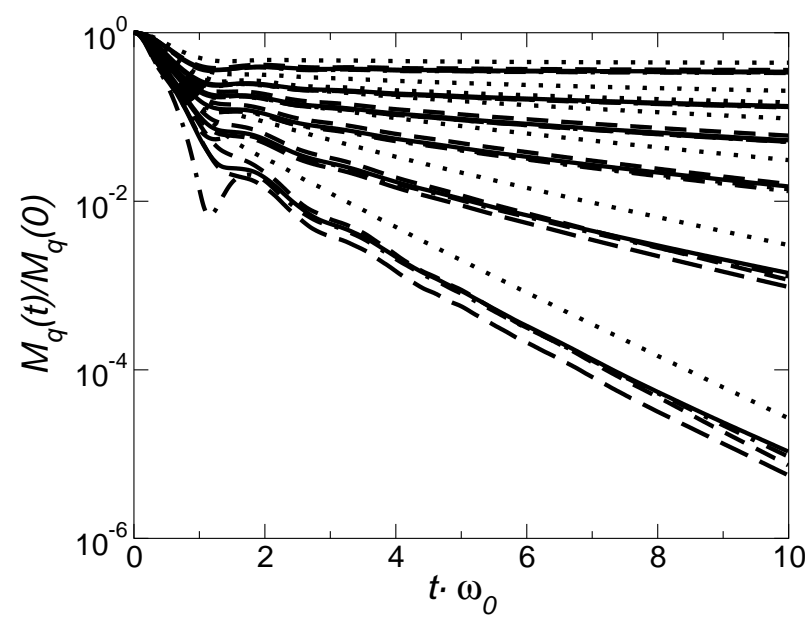

Figure 5. Normalized Memory functions for (from bottom to top) $\eta=0.40, \eta=0.45, \eta=0.49$, $\eta=0.52, \eta=0.55, \eta=0.60$ and for wavenumbers $q \sigma=1$ (dots), 3 (short dashes), 5 (long dashes) 7 (lines), 9 (dash-dots). The maximum of $S(q)$ is at $q \sigma=7$.

In figure 5 we have plotted $M_{q}(t)$ for the densities of figures 3 and 4 , as well as for $\eta=0.60$, which corresponds to the glassy state. It is striking that these functions do not oscillate strongly, 
but decay rather monotonously. This decay can be roughly described according to

$$
M_{q}(t)=A_{1}(q) \mathrm{e}^{-t / \tau_{1}(q)}+A_{2}(q) \mathrm{e}^{-t / \tau_{2}(q)} .
$$
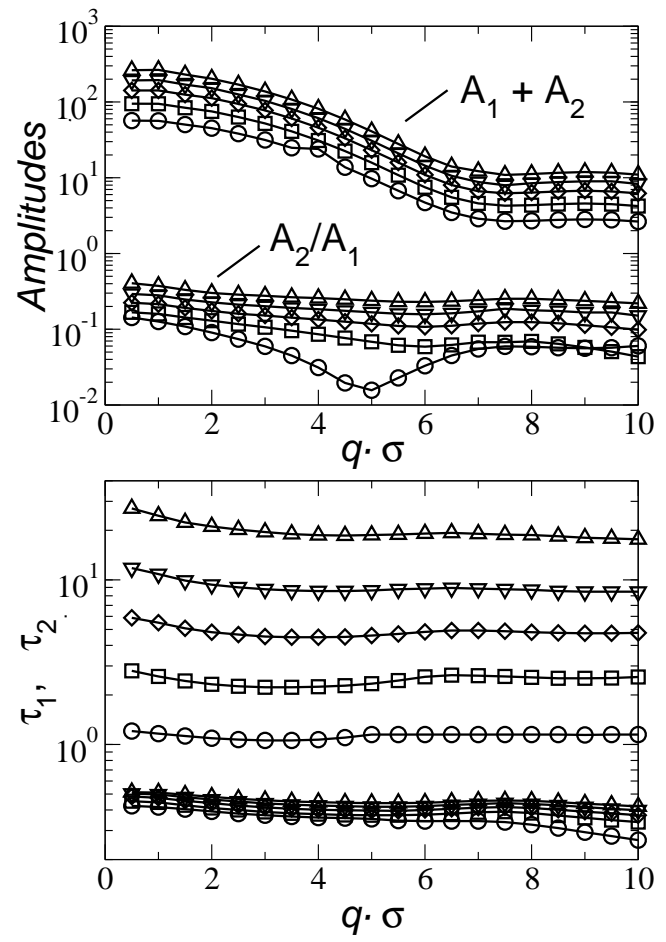

Figure 6. Upper part: Sum and ratio of the amplitudes $A_{2}$ and $A_{1}$. Lower part: Relaxation times $\tau_{2}$ (top) and $\tau_{1}$ (bottom). The packing fraction in all graphs are circles: $\eta=0.40$ squares: $\eta=0.45$ diamonds: $\eta=0.49$ down triangles: $\eta=0.52$ up triangles: $\eta=0.55$.

We have fitted these functions according to equation (13) with amplitudes $A_{1}, A_{2}$, and the relaxation times $\tau_{1}$ and $\tau_{2}$ as $q$-dependent adjustable parameters. The sum of the amplitudes as shown in the upper part of figure 6 is given by the expression

$$
A_{1}+A_{2}=M_{q}(0)=v_{\mathrm{th}}^{2} q^{2} \frac{1}{2 V} \sum_{\mathbf{q}_{1}, \mathbf{q}_{2}}^{\mathbf{q}_{1}+\mathbf{q}_{2}=\mathbf{q}} W_{\mathbf{q q}_{1} \mathbf{q}_{2}}^{2} S\left(q_{1}\right) S\left(q_{2}\right) .
$$

As this function strongly decreases with $q \sigma$ we can conclude that the important relaxation processes have wavelengths larger than an inter-atomic distance. The ratio of the amplitudes, i.e. the relative strength of the two relaxation processes turns out to be almost independent of $q$. The amplitude of the slower relaxation $A 2$ increases with higher packing fractions, indicating the dominance of slow relaxation processes at higher densities. The corresponding relaxation times are plotted in the lower part of figure 6. Surprisingly, there is again almost no $q$-dependence observed. If plotted against packing fraction (figure 7 ), the fast relaxation $\tau_{1}$ turns out to be almost constant with density, while the slow relaxation shows an increase towards higher densities, which can be related to the increase in density discussed earlier.

It is remarkable that the DMCT predicts the existence of two distinct relaxation time regimes with only the static structure factor as an essential input. At densities corresponding to the melting point $(\eta \approx 0.45)$, the ratio $\tau_{1} / \tau_{2}$ is about ten, which is similar to recent experimental findings in simple liquid metals like liquid aluminum [14] and lithium [13], even though the $q$-dependence observed in the experiments is somewhat more complicated. The findings in [13] are corroborated 
in a recent computer simulation on the dynamics of liquid lithium [16], where the data can also be fitted with a memory function of the form (13) with a temperature and $q$ dependence very similar to those given in figure 6 .

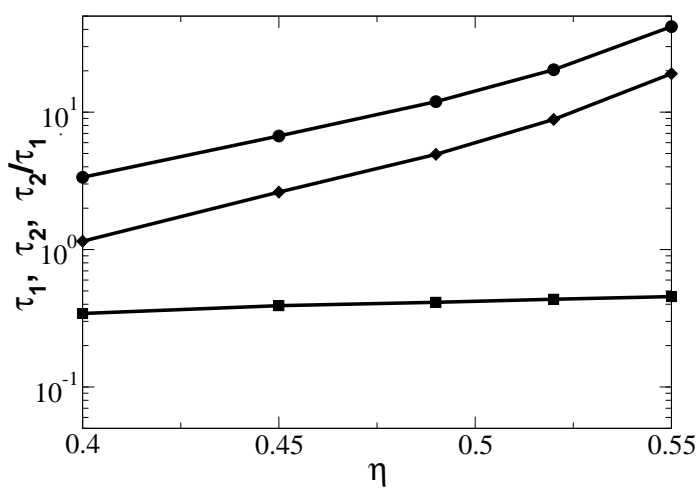

Figure 7. Relaxation times $\tau_{1}$ (squares), $\tau_{2}$ (diamonds) and the ratio $\tau_{2} / \tau_{1}$ (circles) as a function of packing fraction $\eta$ for $q \sigma=7(\approx$ Maximum of $S(q))$.
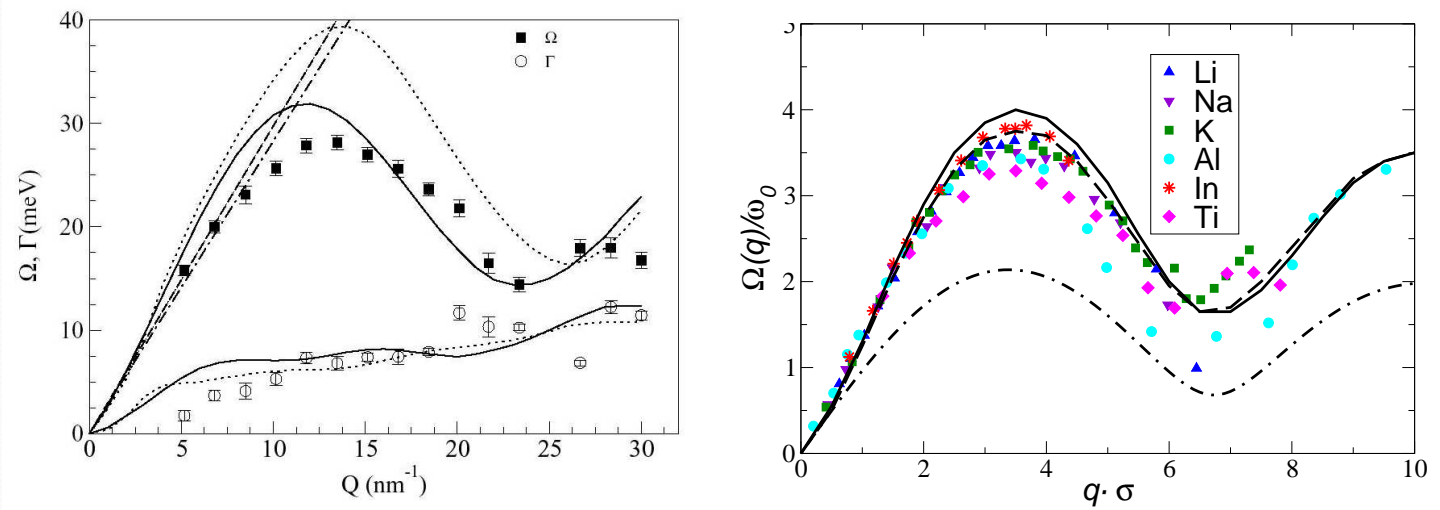

Figure 8. Left: Dispersion of the maxima of the measured current correlation function in liquid $\mathrm{Ti}$ and the linewidth (HWHM) together with MCT results using measured structure factors [26]. Right: dispersion of the maxima of $\tilde{C}(q, \omega)$ of several liquid metals $[5,26,15]$ together with the MCT results for $\eta=0.40$ (dashes) and $\eta=0.45$ (full line). The dash-dotted line is the isothermal dispersion $\Omega_{0}(q)=q \sqrt{S(0) / S(q)}$.

A further quantitative test of the MCT was done recently on the dynamics of liquid titanium [26] (see figure 8a). Instead of the structure factor in equation (8) experimental structure factors were used as input with only the high $q$-range fitted to hard sphere structure factor similar to equation (8). This was necessary, because the compressibility limit $S(q=0)$ in the PY approximations turns out to be about twice as high compared to the experimental values [31]. With equation (3) this would lead to a much lower dispersion curve in the calculations than that observed in the experiment. Using two different experimental structure factors from the literature, the dispersion of the sound modes can be described satisfactorily by the MCT (solid and dotted curves in figure 8a). Note that the uncertainties in the experimental structure factors lead to considerable shifts in the dispersion calculated by MCT. On the other hand, the damping of the modes is well described by both structure factors, independent of the relatively large experimental uncertainty of $S(q)$ at small $q$. In figure $8 \mathrm{~b}$ we have plotted the measured longitudinal dispersions $\Omega(q)$ - plotted in units of $\omega_{0}$ and $\sigma^{-1}$. Firstly it is remarkable that they all collapse into a universal plot. Secondly they almost coincide with the result of MCT (full line: $\eta=0.45$, dashed line: $\eta=0.40$ ). We conclude 
that MCT prediction is correct both from the viewpoint of the scaling and the quantitative value of $\Omega(q)$.

It will be interesting to explore which kind of liquids shows the same scaled dispersion and which properties of liquids lead to a deviation from this law of corresponding states given by MCT. As stated above it remains to be shown, that $\Omega(q)$ stays the same as the density is lowered. Also, to verify the universality of the linewidth and the two-step decay of the memory function, more measurements and simulations are required.

\section{Conclusions}

Using smoothed hard-sphere-Percus-Yevick structure factors as input we have succeeded to obtain a MCT description of the collective dynamics of simple liquids, which essentially depends only on one single parameter, namely the packing fraction $\eta$. This parameter characterizes the density of the liquid relative to its melting point, where it takes usually values near 0.45 . MCT then predicts that the dispersion and damping of the longitudinal sound-like excitations as given by the maximum and half-width of the current correlation spectrum does not essentially depend on $\eta$, i.e. remains the same as in the undercooled limit. The relaxation as described by MCT consists of two distinct relaxation time regimes. The fast relaxation is on microscopic time scales and is almost independent of the density. The slower relaxation can be identified as the alpha-relaxation known from the dynamics close to the glass transition. Even though the coupling to transverse modes is neglected in the theory, it is capable of describing the experimentally observed relaxation times, the dispersion of sound modes and their damping in simple liquid metals.

\section{Acknowledgements}

This work was supported by the US DOE, Office of Science, Office of Basic Energy Science within the framework of the Argonne Theory Institute under contract No. W-31-109-ENG-38.

\section{Appendix: Evaluation of the memory function}

In order to evaluate the sum in (4) we define a vector $\mathbf{Q}$ by

$$
\mathbf{q}_{1}=\frac{\mathbf{q}}{2}+\mathbf{Q}, \quad \mathbf{q}_{2}=\frac{\mathbf{q}}{2}-\mathbf{Q}
$$

We perform the $\mathbf{Q}$ integration in cylinder coordinates $\mathbf{Q}=(\rho, z, \varphi)$ and use $\sum_{\mathbf{Q}} \equiv \frac{V}{(2 \pi)^{3}} \int \mathrm{d} \mathbf{Q}$ :

$$
\begin{aligned}
M_{q}(t) & =v_{\mathrm{th}}^{2} q^{2} \frac{1}{16 \pi^{3} n} \iiint \rho \mathrm{d} \rho \mathrm{d} z \mathrm{~d} \varphi W_{\mathbf{q q}_{1} \mathbf{q}_{2}}^{2} F_{q_{1}}(t) F_{q_{2}}(t) \\
& =v_{\mathrm{th}}^{2} q^{2} \frac{1}{4 \pi^{2} n} \int_{0}^{Q^{*}} Q \mathrm{~d} Q \int_{0}^{Q} \mathrm{~d} z W_{\mathbf{q}_{\mathbf{q}_{1} \mathbf{q}_{2}}^{2}}^{2} F_{q_{1}} F_{q_{2}}(t),
\end{aligned}
$$

where we have used $\rho \mathrm{d} \rho=Q \mathrm{~d} Q$ and the fact that the integrand is even in $z$. With the definitions (A1) we obtain for the inner vertex

$$
W_{\mathbf{q q}_{1} \mathbf{q}_{2}}=\frac{1}{2}\left[n c\left(q_{1}\right)+n c\left(q_{2}\right)\right]+\frac{z}{q}\left[n c\left(q_{1}\right)-n c\left(q_{2}\right)\right]
$$

we now introduce wavenumbers in units of the hard sphere diameter $\sigma: \tilde{\mathbf{q}} \equiv \mathbf{q} \sigma$ yielding

$$
M_{q}(t)=\omega_{0}^{2} \tilde{q}^{2} \frac{1}{24 \pi \eta} \int_{0}^{\tilde{Q}^{*}} \tilde{Q} \mathrm{~d} \tilde{Q} \int_{0}^{\tilde{Q}} \mathrm{~d} \tilde{z} W_{\tilde{\mathbf{q}} \tilde{\mathbf{q}}_{1} \tilde{\mathbf{q}}_{2}}^{2} F_{\tilde{q}_{1}}(t) F_{\tilde{q}_{2}}(t) .
$$




\section{References}

1. Copley J.R.D., Lovesey S., Rep. Prog. Phys., 1975, 38, 461.

2. Egelstaff P.A. An Introduction to the Liquid State, 2nd Edition. Clarendon Press, Oxford 1994.

3. Boon J.P., Yip S., Molecular Hydrodynamics. McGraw Hill. New York, 1980.

4. Hansen J.-P., McDonald I.R. Theory of Simple Liquids, 2nd Edition, Academic Press, Amsterdam 1986.

5. For a recent exhaustive review see Scopigno T., Ruocco G., Sette F., Rev. Mod. Phys., 2005, 77, 881.

6. Wertheim M.S., Phys. Rev. Lett., 1963, 10, 321; Thiele E., J. Chem. Phys., 1963, 39, 474.

7. Chapman S., Cowling T. The Mathematical Theory of Non-Uniform Gases. Cambridge, England, 1952.

8. Protopapas P., Andersen H.C., Parlee N.A.D., J. Chem. Phys., 1973, 49, 15.

9. Scopigno T., Di Leonardo R., Comez L., Baron A.Q.R., Fioretto D., Ruocco G., Phys. Rev. Lett., 2005, 94, 155301.

10. Forster D., Hydrodynamics, Broken Symmetry and Correlation Functions Benjamin, Reading, Mass., 1975.

11. Hubbard J., Beeby J.L., J. Phys. C, 1969, 2, 556.

12. Levesque D., Verlet L., Kurkijärvi J., Phys. Rev. A 7, 1690 (1973).

13. Scopigno T., Balucani U., Ruocco G., Sette F., Phys. Rev. Lett. 85, 4076 (2000).

14. Scopigno T., Balucani U., Ruocco G., Sette F., Phys. Rev. E 63, 011210 (2001).

15. Reichert H., Bencivenga F., Wehinger B., Krisch M., Sette F., Dosch H., Phys. Rev. Lett., 2007, 98, 096104.

16. Scopigno T., Ruocco G., Sette F., Viliani G., Phys. Rev. E, 2002, 66, 031205.

17. de Schepper I., Verkerk P., Well A.A.V., de Graaf L., Phys. Rev. Lett., 1983, 50, 974.

18. Götze W., Lücke M., Phys. Rev. A, 1976, 13, 3822; ibid., 3825

19. Bengtzelius U., Götze W., Sjölander A., J. Phys. C, 1984, 17, 5915.

20. Leutheusser E., Phys. Rev. A, 1984, 29, 2765.

21. Götze W., in Liquids, Freezing and the Glass Transition, Hansen J.-P., Levesque D., Zinn-Justin J., Eds., North-Holland, Amsterdam 1991.

22. Götze W., Sjögren L., Rep. Prog. Phys., 1992, 55, 241.

23. Götze W., Mayr M. R., Phys. Rev. E, 2000, 61, 587.

24. Chong S.-H., Phys. Rev. E, 2006, 74, 031205.

25. Götze W., Lücke M., Phys. Rev. A, 1976, 11, 2173. Bosse J., Götze W., Lücke M., Phys. Rev. A, 1978, 17, 434; ibid., 1978, 17, 447; ibid., 1978, 18, 1176.

26. Said A.H., Sinn H., Alatas A., Burns C.A., Price D.L., Saboungi M.L., Schirmacher W., Phys. Rev. B, 2006, 74, 172202 .

27. Wiebel S., Wuttke J., New J. Phys., 2002, 4, 56.1.

28. Note that this definition of the Fourier-Laplace transform [4] differs from that in got91 by a factor $i$, so that real and imaginary part are interchanged.

29. Horbach J., Kob W., Binder K., Eur. Phys. J. B, 2001, 19, 531.

30. This relation holds only if temperature fluctuations are disregarded, as is done in the present treatment. In the presence of temperature fluctuations $S(q, \omega=0)$ becomes inversely proportional to $q^{2} \lambda$ at small $q$ where $\lambda$ is the thermal conductivity, see Mountain R.D., Rev. Mod. Phys., 1966, 38, 205.

31. Evans R., Schirmacher W., J.Phys., 1978, C11, 2437. 


\title{
Колективна динаміка простих рідин: опис підходом взаємодіючих мод
}

\author{
В.Шірмахер ${ }^{1,2}$, Г.Зінн ${ }^{1,3}$
}

1 Арґонська Національна Лабораторія, Арґон, США

2 Відділ фізики E13, Технічний університет Мюнхена, Ґархінґ, Німеччина

3 Hasylab/DESY, Гамбурґ, Німеччина

Отримано 27 листопада 2007 р., в остаточному вигляді - 12 лютого 2008 р.

Ми використовуємо теорію взаємодіючих мод (ТВМ), що була надзвичайно успішною у врахуванні аномальної релаксаційної поведінки поблизу переходу рідина-скло, для опису динаміки простих (моноатомних) рідин далеко від режиму формування скла. Ми виявили, що динамічний структурний фактор, передбачений з ТВМ, добре узгоджується з експериментальними даними та результатами комп'ютерного моделювання. Функція пам'яті проявляє двоступеневий спад, що часто отримується з експериментальних та комп'ютерних даних. Режим довгочасової релаксації, в якому швидкість релаксації сильно залежить від густини, ідентифікується як $\alpha$ релаксація. При високій густині цей процес приводить до нестабільності з утворенням скла. Короткочасовий спад релаксації майже не залежить від густини.

Ключові слова: динаміка рідин, колективні збурення в рідинах, прості рідини, теорія взаємодіючих мод

PACS: $62.60 .+V, 66.20 .-d$ 
